\title{
Adrenergic control of pinealocyte chondriome - an in vitro study
}

\author{
B. Przybylska-Gornowicz, B. Lewczuk, N. Ziółkowska, M. Prusik \\ Department of Histology and Embryology, Faculty of Veterinary Medicine, \\ University of Warmia and Mazury in Olsztyn, Oczapowskiego 13, 10-714 Olsztyn, Poland
}

\begin{abstract}
Norepinephrine released from sympathetic innervation plays the main role in the regulation of melatonin secretion in mammalian pinealocytes. The present study was conducted for the following reasons: 1) to establish whether the pinealocyte chondriome is controlled by norepinephrine, 2) to determine the effect of adrenergic stimulation on mitochondria, and 3) to characterize adrenoceptors involved in the regulation of the chondriome.

The static organ culture of the pineal gland was used. The explants were incubated for 5 consecutive days in control medium and between 20:00 and 08:00 in medium with the presence of $10 \mu \mathrm{M}$ norepinephrine - adrenergic agonist; isoproterenol - beta-adrenoceptor agonist; cirazoline, methoxamine, M-6364 - alfa ${ }_{1}$ - adrenoceptors agonists or PMA - activator of PKC. The explants were then subjected to ultrastructural examination and morphometric analysis.

The incubation of explants in the presence of norepinephrine or isoproterenol caused a decrease in the relative volume and the numerical density of mitochondria and induced an increase in the percentage of free mitochondria in pinealocytes. Significant changes in these parameters were not observed after treatment with methoxamine, cirazoline, M-6463 and PMA.

The results obtained show that the chondriome of pig pinealocytes is controlled by norepinephrine acting via beta-adrenoceptors. Adrenergic stimulation, repeated for five consecutive days of organ culture, causes a decrease in the number of mitochondria and a shift in the distribution of mitochondria from the form of networks and filaments into the form of single particles. This indicates the intensive remodeling of the mitochondria network, which is closely linked to the metabolic status of the cell.
\end{abstract}

Key words: mitochondria, pinealocyte, pineal, adrenergic, ultrastructure

\section{Introduction}

Pinealocytes, the primary cells of the mammalian pineal gland, are the sites of melatonin synthesis. The noradrenergic innervation originating from the superior cervical ganglia is the most important neuronal input regulating rhythmic synthesis and the release of melatonin. Norepinephrine (NE) influences the activity of pinealocytes through two postsynaptic adrenergic receptors: $\beta_{1}$ and $\alpha_{1}$ (Ho and Chik 1990, Gupta et al. 2005). In all studied mammals, $\beta_{1}$-adrenoceptors are responsible for the nocturnal increase in 
melatonin secretion, while the role of $\alpha_{1}$-adrenoceptors varies markedly between species (Simonneaux and Ribelayga 2003, Lewczuk et al. 2014).

Hitherto, the effect of neurotransmitters regulating melatonin secretion on the ultrastructure of pinealocytes has not been well recognized. Experimental data concerning the changes in pinealocyte morphology under various physiological and experimental conditions showed that mitochondria, among several examined organelles, appear to be one of the most dynamic cytoplasmic structures (Karasek et al. 2002, Nowicki et al. 2002, Redondo et al. 2003, Kus et al. 2004, Zielińska et al. 2006). However, only simple quantitative assessments of mitochondria have been included in previous pineal studies, and more detailed analyses are needed.

Mitochondria have both general roles and cell specific functions. In pinealocytes, they are involved in the indole metabolism as sites of tryptophan hydroxylation (Hori et al. 1976) and amine oxidative deamination (Przybylska et al. 1994a) and are also involved in calcium turnover (Lewczuk et al. 1994). It is now well known that mitochondria continuously change their volume and shape through frequently occurring fusion, fission, and movements (Rube and van der Bliek 2004). These events are regulated by poorly recognized, complex mechanisms (Benard and Karbowski 2009, Palmer et al. 2011, Van Laar and Berman 2013). Mitochondria are also able to vary their morphology between mitochondrial networks and fragmented disconnected arrays (Kuzmicic et al. 2011). Generally, they exist in the following two interconverting forms: small isolated particles or extended filaments, networks or clusters (Skulachev 2001). It is assumed that a single mitochondrion represents a transient fraction of the chondriome (Bereiter-Hahn 2008). The mitochondrial distribution in the cells also changes (Dedov et al. 2000, Mironov 2007). Alterations in mitochondrial morphology (i.e., volume, distribution and aggregation) are associated with changes in cell metabolism (Larsen et al. 2012, Elgass et al. 2013, Chistiakov et al. 2014). The dynamics of mitochondria are crucial in the maintenance of cellular homeostasis and adaptation (Otera and Mihara 2011, Otera et al. 2013). The structural and functional heterogeneity of mitochondria creates the possibility that subpopulations of these organelles can carry out diverse processes within different areas of the cell (Collins et al. 2002).

Our studies on the morphology of the pig pineal gland showed mitochondria as structures of pinealocytes, which are highly sensitive to various experimental manipulations. Changes in the relative volume of mitochondria were observed after administration of progesterone, progesterone with estradiol (Wy- rzykowski et al. 1990), melatonin (Przybylska et al. 1994b, Lewczuk and Przybylska-Gornowicz 2000), p-chlorophenylalanine and amiflamine (Przybylska et al. 1994b). The bilateral ovariectomy of sows also caused modifications in mitochondria (Wyrzykowski et al. 1992). Similarly, our data indicate that the volume of mitochondria is controlled by environmental light conditions (Lewczuk and Przybylska-Gornowicz 2000). Until now, it has not been determined whether $\mathrm{NE}$, the main driving force behind melatonin synthesis (Ho and Chik 1990), exerts any influence on mitochondria in pig pinealocytes.

The role of adrenergic receptors in mitochondrial biogenesis and function has been the subject of many investigations performed on various cell types (Chiang et al. 2012, Wills et al. 2012, Branco et al. 2013, Peterson et al. 2013, Pennanen et al. 2014). The aim of the present study was to characterize the effect of $\mathrm{NE}$ as well as $\beta$ - and $\alpha_{1}$-adrenergic agonists on mitochondria in pig pinealocytes in organ culture. Our study is the first to provide a detailed analysis of changes in the chondriome of mammalian pinealocytes in response to adrenergic stimulation. Although it has well been established that the mitochondrial population exists as a three-dimensional network, two-dimensional imaging using transmission electron microscopy is still regarded as the golden standard for quantitative analysis of mitochondria (Larsen et al. 2012), and this method was used in the present study.

\section{Materials and Methods}

\section{Animals and pineal glands}

Female crossbred pigs, 12 individuals, $95 \pm 5$ days of age, weighing $30-35 \mathrm{~kg}$ were purchased 14 days before the experiments from a commercial piggery (with natural length of day) and used in the investigation. The animals were kept in a room, in which natural lighting from windows (in April) was supplemented during the day with fluorescent illumination with a light intensity of $500 \mathrm{~lx}$ at the level of the animals' heads. The fluorescent illumination was automatically turned on at 06:00 and turned off at 20:00. The gilts had free access to water and were fed twice daily (08:00 and 14:00) with standard food.

The gilts were slaughtered between 10:00 and 10:30 a.m. The pineal glands (4 in each experiment) were removed under sterile conditions as quickly as possible and placed in Hanks' Balanced Salts Solution (Sigma, USA) with penicillin (1000 U/ml, Sigma-Aldrich, USA), streptomycin (100 $\mu \mathrm{g} / \mathrm{ml}$, Sigma-Aldrich, USA) and amphotericin B $(2.5 \mu \mathrm{g} / \mathrm{ml}$, Sigma-Aldrich, USA). 
Table 1. Treatment schedule in the first experiment.

\begin{tabular}{|c|c|c|c|c|c|c|c|c|c|c|}
\hline & \multicolumn{2}{|c|}{ Day 1} & \multicolumn{2}{|c|}{ Day 2} & \multicolumn{2}{|c|}{ Day 3} & \multicolumn{2}{|c|}{ Day 4} & \multicolumn{2}{|c|}{ Day 5} \\
\hline & $\begin{array}{c}08: 00 \\
-20: 00 \\
\end{array}$ & $\begin{array}{c}20: 00 \\
-08: 00 \\
\end{array}$ & $\begin{array}{c}08: 00 \\
-20: 00 \\
\end{array}$ & $\begin{array}{c}20: 00 \\
-08: 00\end{array}$ & $\begin{array}{c}08: 00 \\
-20: 00 \\
\end{array}$ & $\begin{array}{c}20: 00 \\
-08: 00\end{array}$ & $\begin{array}{c}08: 00 \\
-20: 00 \\
\end{array}$ & $\begin{array}{c}20: 00 \\
-08: 00\end{array}$ & $\begin{array}{c}08: 00 \\
-20: 00 \\
\end{array}$ & $\begin{array}{c}20: 00 \\
-24: 00 \\
\end{array}$ \\
\hline Group C & $\mathrm{C}$ & $\mathrm{C}$ & $\mathrm{C}$ & $\mathrm{C}$ & $\mathrm{C}$ & $\mathrm{C}$ & $\mathrm{C}$ & $\mathrm{C}$ & $\mathrm{C}$ & $\mathrm{C}$ \\
\hline Group NE-1 & $\mathrm{C}$ & $\mathrm{NE}$ & $\mathrm{C}$ & $\mathrm{NE}$ & $\mathrm{C}$ & $\mathrm{NE}$ & $\mathrm{C}$ & $\mathrm{NE}$ & $\mathrm{C}$ & $\mathrm{NE}$ \\
\hline Group NE-2 & $\mathrm{C}$ & $\mathrm{C}$ & $\mathrm{C}$ & $\mathrm{C}$ & $\mathrm{C}$ & $\mathrm{C}$ & $\mathrm{C}$ & $\mathrm{NE}$ & $\mathrm{NE}$ & $\mathrm{NE}$ \\
\hline
\end{tabular}

Explanations: $\mathrm{C}-$ incubation in control medium, NE - incubation in medium with $10 \mu \mathrm{M}$ of $\mathrm{NE}$

Table 2. Administration schedule of drugs in the second experiment.

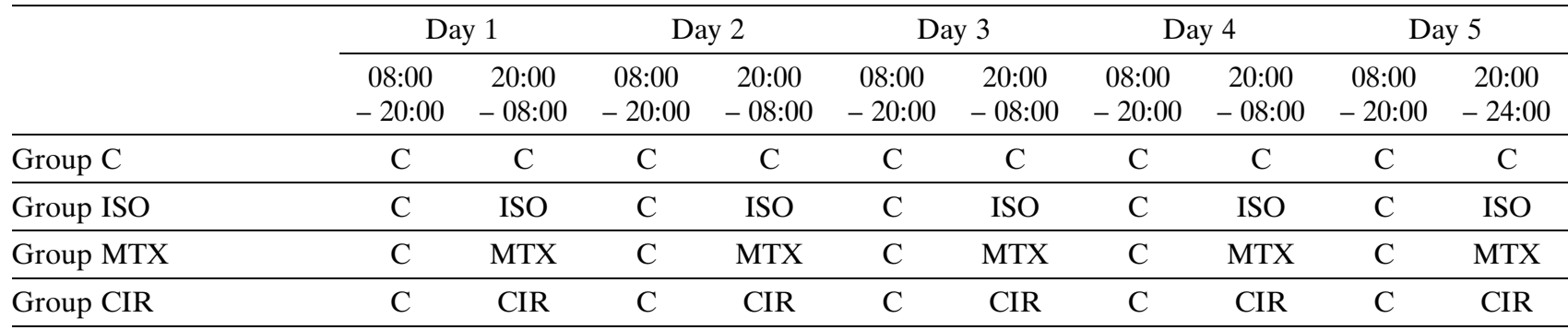

Explanations: $\mathrm{C}$ - incubation in control medium, ISO - incubation in medium with $10 \mu \mathrm{M}$ of isoproterenol, MTX - incubation in medium with $10 \mu \mathrm{M}$ of methoxamine, CIR - incubation in medium with $10 \mathrm{mM}$ of cirazoline.

Table 3. Administration schedule of drugs in the third experiment.

\begin{tabular}{lccccccccccc}
\hline & \multicolumn{2}{c}{ Day 1 } & \multicolumn{2}{c}{ Day 2 } & \multicolumn{2}{c}{ Day 3 } & \multicolumn{2}{c}{ Day 4 } & \multicolumn{2}{c}{ Day 5 } \\
\cline { 2 - 13 } & $08: 00$ & $20: 00$ & $08: 00$ & $20: 00$ & $08: 00$ & $20: 00$ & $08: 00$ & $20: 00$ & $08: 00$ & $20: 00$ \\
& $-20: 00$ & $-08: 00$ & $-20: 00$ & $-08: 00$ & $-20: 00$ & $-08: 00$ & $-20: 00$ & $-08: 00$ & $-20: 00$ & $-24: 00$ \\
\hline Group C & C & C & C & C & C & C & C & C & C & C \\
\hline Group PMA & C & PMA & C & PMA & C & PMA & C & PMA & C & PMA \\
\hline Group M-6463 & C & M-6463 & C & M-6463 & C & M-6463 & C & M-6463 & C & M-6463 \\
\hline
\end{tabular}

Explanations: $\mathrm{C}$ - incubation in control medium, PMA - incubation in medium with $10 \mu \mathrm{M}$ of PMA, M-6463 - incubation in medium with $10 \mu \mathrm{M}$ of M-6463.

All experimental procedures on animals were performed in accordance with Polish and EU law. They were approved by the Local Ethics Committee for Experiments on Animals in Olsztyn.

\section{Organ culture}

The pineal glands were transported to the cell culture lab within less than 3 minutes. Each pineal gland was divided into several explants, which were randomly assigned to the control and experimental groups. These tissue pieces were placed on the meshes of tissue culture inserts (Netwell $74 \mu \mathrm{m}$, Costar, USA) in a 12-well culture plate containing $0.5 \mathrm{ml}$ BGJb Fitton-Jackson modification medium (Life Technologies, USA) with the addition of $5 \%$ calf serum (Sigma-Aldrich, USA), penicillin (100 U/ml, Sigma-Aldrich, USA), streptomycin (100 $\mu \mathrm{g} / \mathrm{ml}$, Sigma-Aldrich) and amphotericin B $(0.25 \mu \mathrm{g} / \mathrm{ml}$, Sigma-Aldrich). The explants were incubated in a humidified atmosphere of $80 \%$ oxygen and $5 \%$ carbon dioxide at $37^{\circ} \mathrm{C}$ in a Kendro BB6060 incubator (Germany). The medium was changed twice daily (at 08:00 and 20:00) by transferring the inserts to a new culture plate with medium which had been pre-incubated, under the conditions described above, for one hour.

\section{Experimental protocols}

The experiments were designed in a way that ensures: 1) complete degeneration of the sympathetic nerve endings and elimination of endogenous $\mathrm{NE}$ from the pineal tissue due to the five-day-long culture period, 2) exposure of explants to adrenergic agonists exclusively during subjective night, which overlaps with the time of physiologically occurring release of 
$\mathrm{NE}$ from the intra-pineal nerve endings, 3) interrupted exposition of explants to agonists (in 12hr-long cycles) to reduce a large down-regulation of receptors, 4) treatment of explants with agonists each day to prevent a large upregulation of receptors. Moreover, repeated exposure to experimental factors during consecutive days usually results in partially additive effects, which facilities the detection of morphological alternation. An exception to this concept was the protocol of group NE-2, in experiment I, which was used to check the effect of single, one-day-long stimulation with NE performed after 96 hours of preincubation period.

\section{Experiment 1}

The tissue pieces were assigned to three groups $(n=4)$. The explants of the control group (C) were incubated in the control medium during the whole experiment, and the explants of groups NE-1 and NE-2 were incubated in the control medium or medium with $10 \mu \mathrm{M}$ NE (Sigma-Aldrich, USA) in accordance with the schedule presented in Table 1. At the end of the experiment (at 24:00, day 5), the explants were removed from the tissue culture inserts, fixed, and prepared for ultrastructural investigations.

\section{Experiment 2}

The tissue pieces were assigned to four groups. The explants of the control group (C) were incubated in the control medium during the whole experiment. The explants of experimental groups were incubated by turns, 12 hours in the control medium and 12 hours in the medium with adrenergic agonists (all from Tocris, UK) as follows: group ISO - with $10 \mu \mathrm{M}$ isoproterenol, group MTX - with $10 \mu \mathrm{M}$ methoxamine and group CIR - with $10 \mu \mathrm{M}$ cirazoline, according to the schedule presented in Table 2. On the last day of culture, the tissue pieces were removed (at 24:00) from the tissue culture inserts and were prepared for observation in the transmission electron microscope.

\section{Experiment 3}

The tissue pieces were assigned to three groups. The explants of the control group (C) were incubated in the control medium for the whole experiment, and the pineal of groups PMA and M-6463 were incubated by turns in the control medium and the medium with $10 \mu \mathrm{M}$ phorbol 12-myristate 13-acetate (PMA, Sigma-Aldrich, USA) or $10 \mu \mathrm{M}$ M-6463 (Tocris, UK), according the schedule presented in Table 3. After the experiments, the tissue pieces were prepared for ultrastructural investigations.

\section{Ultrastructural investigations}

Explants were immersion fixed $\left(2\right.$ hours, $\left.4^{\circ} \mathrm{C}\right)$ in a mixture of $2.5 \%$ glutaraldehyde and $1 \%$ paraformaldehyde in $0.2 \mathrm{M}$ phosphate buffer ( $\mathrm{pH} 7.4$ ), washed, postfixed in $2 \%$ osmium tetroxide for 2 hours, and embedded in Epon 812. Ultrathin sections, stained with uranyl acetate and lead citrate, were examined with a Tecnai 12 Spirit G2 BioTwin transmission electron microscope (FEI, USA).

For quantitative studies, 30 electronograms at a magnification of $8000 \mathrm{x}$ were taken from each explant using a modified systematic random sampling (Weibel 1979). A point count analysis (Weibel 1979) was employed, as characterized in detail in our previous work (Lewczuk and Przybylska-Gornowicz 1997), to estimate the relative volume (expressed as the percent of cytoplasm of pinealocyte perikaryon) and the numerical density of mitochondria (expressed as the number of mitochondria per $\left.60 \mu \mathrm{m}^{2}\right)$. The percentages of mitochondria occurring in the form of small isolated particles or complexes (networks and extended filaments) were also determined.

\section{Statistical analysis}

The morphometric data were analyzed using one-way analysis of variance followed by the Duncan Test as a post-hoc procedure. Statistical analyses were performed using Statistica 10.0 software (StatSoft, USA).

\section{Results}

The structure of the examined explants was well-preserved and only single degenerating or dead cells were observed. From a qualitative point of view, the ultrastructure of cultured pinealocytes exhibited the typical features of these cells in the domestic pig, described in detail in our previous works (Lewczuk and Przybylska Gornowicz 1997, 2000a). Briefly, the pinealocyte cytoplasm showed the presence of short cisterns of rough endoplasmic reticulum, small dictyosomes of the Golgi apparatus, lysosomes, mitochondria, numerous membrane bound dense bodies, and microtubules (Fig. 1). Mitochondria occurred as randomly distributed, isolated particles or formed complexes such as filaments or networks (Fig. 1). 

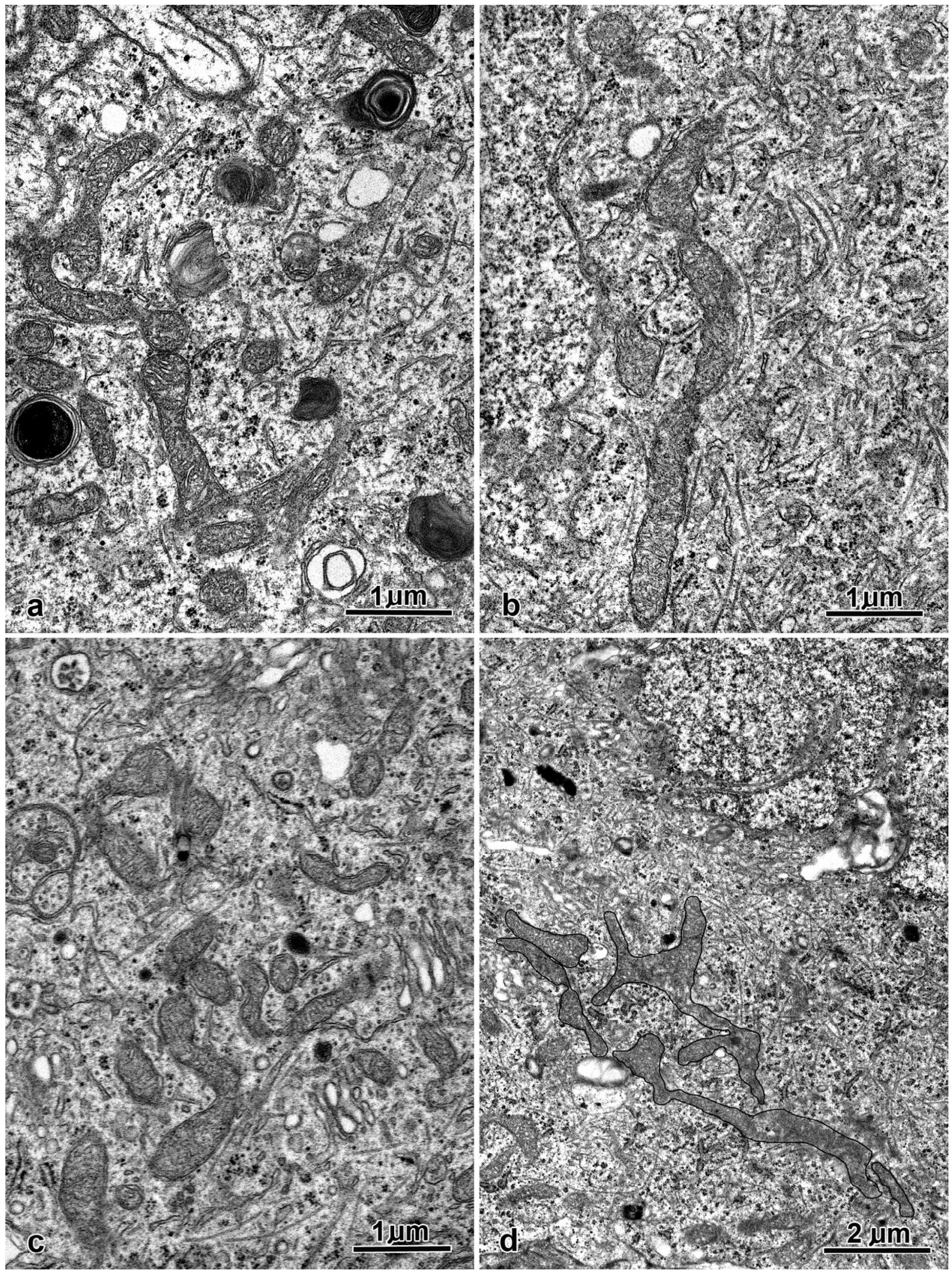

Fig. 1. The ultrastructure of pinealocytes in explants of pig pineal glands. Note the various forms of mitochondria: a) A network and single particles of mitochondria close to the network (experiment 1, group C); b) Mitochondria forming an extended filament. Note the clearly visible site of mitochondria fusion (experiment 1, group NE-1); c) Small networks and single particles of mitochondria in the neighborhood of the dictyosomes of Golgi apparatus (experiment 2, group ISO); d) A large network of mitochondria. The membranes of individual elements forming the network have been cut at different angles, which resulted in the poor visibility of the mitochondrial outlines. Therefore, the contours of mitochondria forming the network were outlined by a black line (experiment 3 , group $\mathrm{C}$ ). 

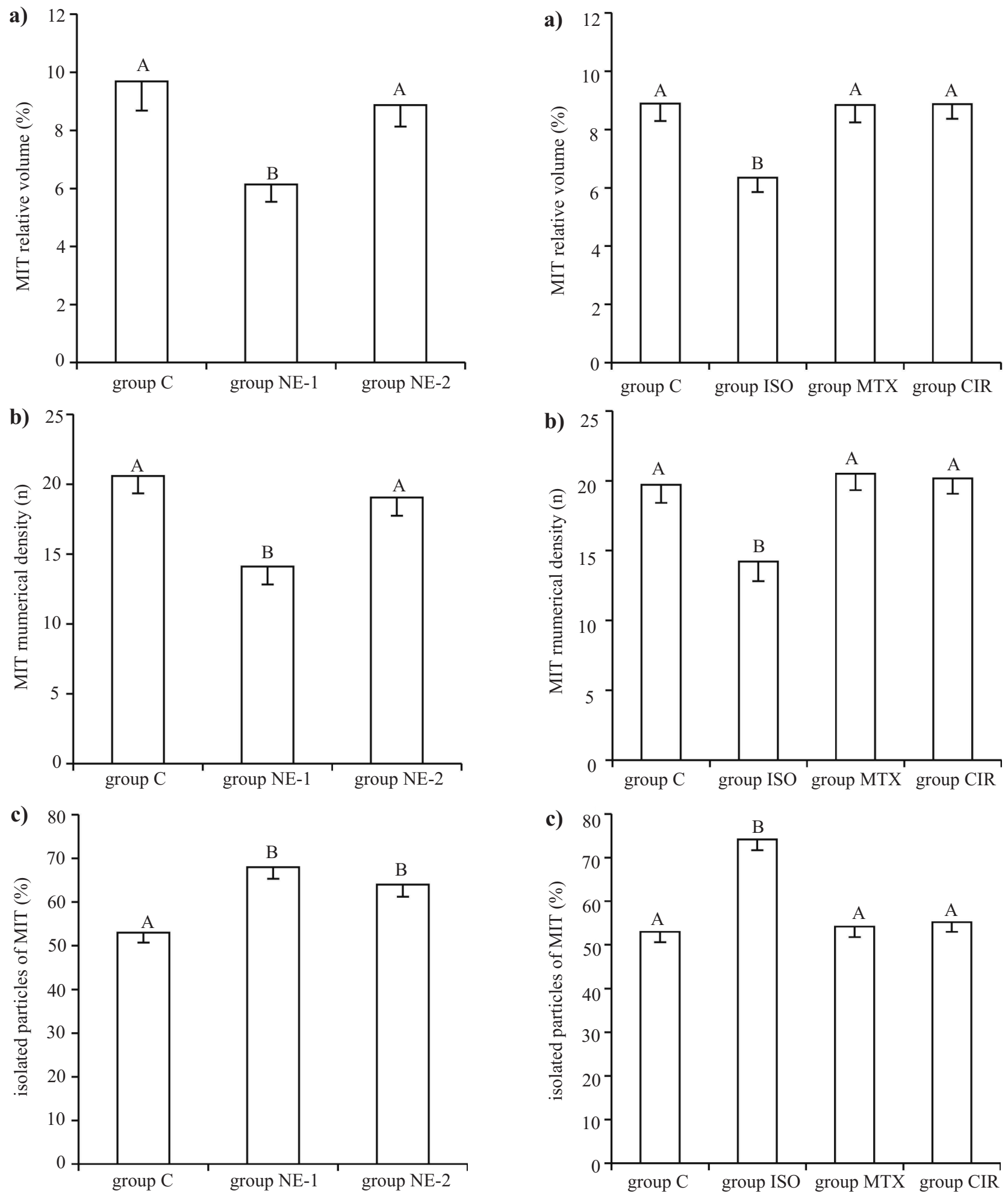

Fig. 2. Results of morphometric analysis of the chondriome in pinealocytes in experiment 1: a) Relative volume of mitochondria in the pinealocyte perikarya; b) Numerical density of mitochondria in the pinealocyte perikarya; c) Percentage of mitochondria occurring as isolated particles in pinealocyte perikarya. Values marked with different letters are significantly different at $\mathrm{p} \leq 0.05$ from each other. The explants were incubated in the control medium during the whole experiment (group C) or exposed to NE on days $1-5$ (group NE-1) or exclusively on day 5 (group NE-2), according to the schedule presented in Table 1.

Fig. 3. Results of morphometric analysis of the chondriome in pinealocytes in experiment 2: a) Relative volume of mitochondria in the pinealocyte perikarya; b) Numerical density of mitochondria in the pinealocyte perikarya; c) Percentage of mitochondria occurring as isolated particles in pinealocyte perikarya. The values marked with different letters are significantly different at $\mathrm{p} \leq 0.05$ from each other. The explants were repeatedly exposed to isoproterenol (group ISO), metoxamine (group MTX), and cirazoline (group CIR) according to the schedule presented in Table 2 . The explants of group $\mathrm{C}$ served as control. 

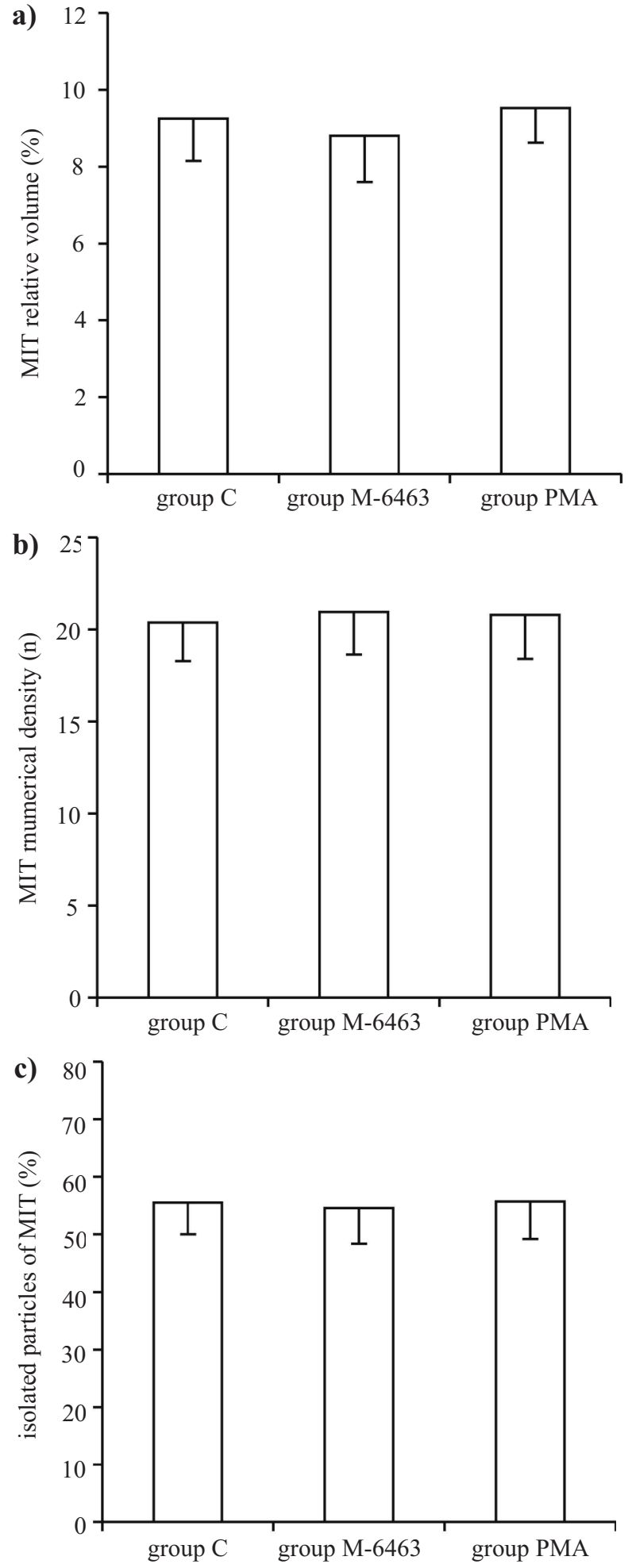

Fig. 4. Results of morphometric analysis of the chondriome in pinealocytes in experiment 3: a) Relative volume of mitochondria in the pinealocyte perikarya; b) Numerical density of mitochondria in the pinealocyte perikarya; c) Percentage of mitochondria occurring as isolated particles in pinealocyte perikarya. The explants were incubated in the control medium (group C) or repeatedly exposed to M-6463 (group M-6463) or PMA (group PMA) according the schedule presented in Table 3 .

\section{Experiment 1}

The repeated treatment of explants with NE for a period of 5 days resulted in a significant decrease in the relative volume and the numerical density of mitochondria in pinealocytes, compared to the control explants (Fig. 2a,b). In contrast, the single, 28-hour-long incubation of the explants with NE did not induce significant changes in the relative volume and the numerical density of mitochondria (Fig. 2a,b).

The percent of mitochondria occurring in the form of isolated particles was significantly higher in pinealocytes of both NE-treated groups than the control group (Fig. 2c).

\section{Experiment 2}

The relative volume and the numerical density of mitochondria in pinealocytes were significantly lower in the group of explants treated with isoproterenol than in the control group and the groups incubated in the presence of methoxamine and cirazoline (Fig. 3a,b).

The percent of mitochondria in a form of isolated particles in pinealocytes was significantly higher in the isoproterenol-treated group than in the other groups (Fig. 3c). The treatment with methoxamine and cirazoline did not result in significant changes in either the relative volume or the numerical density of mitochondria in the occurrence of these organelles in the form of isolated particles (Fig. 3a,b,c).

\section{Experiment 3}

The treatment with M-6463 did not result in statistically significant changes in the relative volume and the numerical density of pinealocyte mitochondria in comparison with the control group (Fig. 4a,b). Furthermore, no significant changes were found in the creation of complex forms by pinealocyte mitochondria in response to treatment with M-6463 (Fig. 4c).

PMA treatment also caused no significant changes in the analyzed parameters of the pinealocyte chondriome (Fig. 4a,b,c).

\section{Discussion}

The results of previous experiments, performed in vivo, demonstrated a close relationship between light conditions and pinealocyte mitochondria, which suggests an involvement of the adrenergic input in the regulation of these organelles (Lewczuk and Przybylska-Gornowicz 2000a, Redondo et al. 2003, 
Kus et al. 2004, Lewczuk et al. 2004). The exposition of pigs to continuous, 14-day-long illumination resulted in a decrease in the relative volume of mitochondria (Lewczuk and Przybylska-Gornowicz 2000a). On the other hand, the exposure to continuous darkness lead to an increase in the relative volume of mitochondria (Lewczuk and Przybylska-Gornowicz 2000a). In rats kept in constant darkness, the number of mitochondria increased, while in constant light, their number decreased (Kus et al. 2004). Concerning diurnal changes, in gilts kept under a $12 \mathrm{hr}$. light: $12 \mathrm{hr}$. dark cycle, the relative volume of mitochondria was significantly higher in pigs slaughtered at 14:00 than at 02:00 (Lewczuk et al. 2004). In contrast, in sheep pinealocytes, the relative volume of mitochondria was significantly greater in animals killed at night (Redondo et al. 2003). Seasonal changes in the amount of mitochondria have been observed in pinealocytes of the domestic pigeon (Madhu and Manna 2010). The results of pharmacologic studies are less conclusive. The administration of amiflamine (MAO-A inhibitor), a drug which increases the content of endogenous NE (Oxenkrug et al. 1991), to immature pigs increased the relative volume of mitochondria in pinealocytes (Przybylska et al. 1994), whereas a similar treatment with clorgyline (another MAO-A inhibitor) has no effect on the relative volume of mitochondria in these cells (Lewczuk and Przybylska-Gornowicz 1997). Changes in mitochondria in pig pinealocytes were not observed after the intravenous administration of isoproterenol, dobutamine, propranolol, and $\alpha$-methyl-p-tyrosine (Lewczuk and Przybylska-Gornowicz 1997).

The data from the in vivo experiments, described above, raise a question about the factors and mechanisms that control mitochondria in mammalian pinealocytes. In view of this, in the present study, we analyzed the role of adrenergic input in the regulation of the pinealocyte chondriome by testing the effects of $\mathrm{NE}$ and selective agonists of adrenergic receptors in the static organ culture of pig pineal explants. The data show significant decreases in the relative volume and the numerical density of mitochondria in pig pinealocytes as a result of the repeated, 12-hour-long treatment with NE, performed over the course of five consecutive days. However, the single, 28-hour long incubation with NE caused no significant changes in the chondriome parameters, which indicates the complex nature of the analyzed processes. The significant decreases in the relative volume and the numerical density of mitochondria were also observed after the treatment with isoproterenol, a very potent beta-agonist. In contrast, no significant changes in both measures of the chondriome were observed after the incubation in the presence of $\alpha_{1}$-adrenoceptor agonists methoxamine, cirazoline and M-6463. Moreover, in treatment with
PMA, an activator of protein kinase $\mathrm{C}$, the enzyme involved in the alpha ${ }_{1}$ adrenoceptor signal transduction pathway was also ineffective. It is worth noting that the adrenergic stimulation induced no changes to the average size of mitochondria, as the proportions of the relative volume to the numerical density were similar in the control and experimental groups (data not shown). Therefore, it is possible to conclude that the number of mitochondria in pig pinealocytes is controlled by $\mathrm{NE}$ via stimulation of beta-adrenoceptors. The decrease in the number of mitochondria observed in our study may come as a result of their higher turnover or the inhibition of their formation (Tomanek 1989, Dhillon and Schulte 2011).

Mitochondria have been reported to be highly dynamic and mobile (Müller et al. 2005, Larsen et al. 2012). The plasticity of the amount and structure of mitochondria is a well-known phenomenon (Perkins and Ellisman 2007, Dhillon and Schulte 2011, Przybylska-Gornowicz et al. 2012, Escobar-Henriques and Anton 2013). The processes of mitochondrial biogenesis, fusion and fission could be involved in various important cell functions. These phenomena are associated with changes in cell metabolism, cell development, and cell death. They are also involved in the transmission of signaling messengers and the exchange of metabolites within the cell (Chada and Hollenbeck 2003, Kuzmicic et al. 2011, Pennanen et al. 2014). Changes in mitochondrial morphology can consequently affect the functioning of the respiratory chain, the regulation of the mitochondrial permeability transition pore, and the interaction with other organelles (Piquereau et al. 2013). Mitochondrial dysfunction would thus give rise to predictable defects in cells and tissues (Chan 2006, Sasaki 2010). Several pathologies have been associated with perturbations in the cellular machinery which coordinate the biogenesis and transformations of mitochondria (Duchen 2004, Elgass et al. 2012, Otera et al. 2013). The structural forms of chondriome strictly depend on the amount of these organelles. High density of mitochondria in some cells, or within regions of the cell, results in the formation of clusters, networks or̀ mitochondrial' filaments and low density means occurrence of mitochondria as isolated particles (Mironov 2006, 2007, Perkins and Ellisman 2011). Furthermore, mitochondrial fission not only enables the proper distribution of mitochondria but also contributes to the selective removal of damaged organelles (Escobar-Henriques and Anton 2013). The maintenance of mitochondria in mammalian cells is a complex process regulated mainly by the GTPase family of proteins (Otera and Mikara 2011, Reddy et al. 2011).

Considering the significance of mitochondrial fusion and fission, the present study investigated, for 
the first time in pinealocytes, the effect of adrenergic stimulation on the occurrence of mitochondria in the form of networks and filaments or as isolated particles. The obtained results show that NE treatment caused a decrease in the percentage of mitochondria-formed networks/filaments and an increase in the percentage of free mitochondria when compared to the control group. Notably, this phenomenon concerned both groups of explants treated with the NE. Similar changes were also found after the incubation of the pineal explants in the medium with isoproterenol, which indicates an important role of beta-adrenoceptors in the regulation of mitochondrial fission and fusion. The lack of significant effects of methoxamine, cirazoline and M6463 on the percentages of single mitochondria particles and mitochondria-formed networks or filaments excluded the involvement of $\alpha$-adrenoceptors as essential components of this regulatory mechanism.

Our data mostly comply with the results obtained in the research of other tissues that show adrenergic regulation of the chondriome in various cell types. It has been demonstrated that NE promotes mitochondrial fission leading to a decrease in the mitochondrial mean volume and an increase in the relative number of mitochondria per cell in rat neonatal cardiomyocytes (Pennanen et al. 2014). However, in contrast to pig pinealocytes, NE acts on cardiomyocytes through alpha $_{1}$-adrenoceptors. The in vivo administration of clenbuterol, a beta $a_{2}$-adrenoceptor agonist, induced prominent ultrastructural changes in mitochondria in the skeletal muscle of mice (Sundal and Sharma 2007). Another agonist of beta $_{2}$-adrenoceptors, formoterol, stimulated mitochondrial biogenesis in cultured adult feline cardiomyocytes (Wills et al. 2012) and rabbit renal tubule cells (Wills et al. 2012, Peterson et al. 2013). Interestingly, isoproterenol and $\mathrm{NE}$ were ineffective in these in vitro models (Wills et al. 2012, Peterson et al. 2013). It has been demonstrated that the betaadrenoceptor pathway enhances mitochondrial function in human neural stem cells (Chiang 2012). Notwithstanding, betaadrenergic stimulation does not influence the mitochondrial biogenesis in the skeletal muscle in the human (Robinson et al. 2010). In summary, the literature data and our results showed that adrenergic input plays an important role in the regulation of the chondriome in mammals, but the receptors and mechanisms involved in these processes differ between cell types and species.

Individual particles of mitochondria are highly dynamic and the increase in their participation in comparison to networks/filaments may be interpreted as a sign of chondriome structural reconstruction (Arnold et al. 2011). Mitochondrial dynamics are responsible for the reaction of mitochondria to functional require- ments and result in changes in intracellular distribution of these organelles (Chada and Hollenbeck 2003, Bereiter-Hahn et al. 2008). The elevation in the percentage of individual particles of mitochondria noted by us as the result of treatment of pinealocytes with NE or isoproterenol indicates an increase in mitochondrial dynamics. Comparison of the current results with those concerning the secretion of melatonin in the pig pinealocyte in vitro suggests the potential role of mitochondrial dynamics in the synthesis and secretion of melatonin. Incubation of the pig pineal explants in the presence of $\mathrm{NE}$ at a concentration of $10 \mu \mathrm{M}$ (identical with the one used by us in the present experiments) caused a 4-5-fold increase in melatonin secretion. A significant increase in melatonin secretion was also observed in the presence of isoproterenol; however, no changes in the melatonin output were noted in response to the treatment with methoxamine and cirazoline at a concentration of $10 \mu \mathrm{M}$ (Lewczuk 2002).

In conclusion, the obtained results demonstrate that the mitochondria in pig pinealocytes are under adrenergic control. The beta-adrenoceptors appear to play a major role in this regulation. Adrenergic stimulation caused remodeling of the mitochondrial network, which is known to be related to the metabolic status of the cell. Further studies are required to clarify the relationship between the organization of chondriome and the physiology of pinealocytes.

Ethical approval: All applicable international, national, and institutional guidelines for the care and use of animals were followed. The experimental protocol including animal euthanasia was approved by the Local Ethical Commission for Experiments on Animals at the University of Warmia and Mazury in Olsztyn.

\section{References}

Arnold B, Cassady SJ, VanLaar VS, Berman SB (2011) Integrating multiple aspects of mitochondrial dynamics in neurons: age related differences and dynamic changes in a chronic rotenone model. Neurobiol Dis 41: 189-200.

Bereiter-Hahn J, Voth M, Mai S, Jendrach M (2008) Structural implications of mitochondrial dynamics. Biotechnol J 3: 765-780.

Bernard G, Karbowski M (2009) Mitochondrial fusion and division: Regulation and role in cell viability. Semin Cell Dev Biol 20: 365-374.

Branco AF, Sampaio SF, Wieckowski MR, Sardao VA, Oliveira PJ (2013) Mitochondrial disruption occurs downstream from $\beta$-adrenergic overactivation by isoproterenol in differentiated, but not undifferentiated $\mathrm{H} 9 \mathrm{c} 2$ cardomyoblasts: differential activation of stress and survival pathways. Int J Biocem Cell Biol 45: 2379-2391.

Chada SR, Hollenbeck PJ (2003) Mitochondrial movement and positioning in axons: the role of growth factor signaling. J Exp Biol 206: 1985-1992. 
Chan DC (2006) Mitochondria: dynamic organelles in disease, aging and development. Cell 125: 1241-1252.

Chiang MC, Lin H, Cheng YC, Yen CH, Huang RN, Link $\mathrm{KH}$ (2012) $\beta$-adrenoceptor pathway enhances mitochondrial function in human neural stem cells via rotary cell culture system. J Neurosci Methods 15: 130-136.

Chistiakov DA, Sobenin IA, Revin VV, Orekhov AN, Bobryshev YV (2014) Mitochondrial aging and age-related dysfunction of mitochondria. Biomed Res Int 2014: 238463.

Collins TJ, Berridge MJ, Lipp P, Bootman MD (2002) Mitochondria are morphologically and functionally heterogeneous within cells. EMBO J 21: 1616-1627.

Dedov VN, Armati PJ, Roufogalis BD (2000) Three-dimensional organization of mitochondrial clusters in regenerating dorsal root ganglion (DRG) neurons from neonatal rats: evidence for mobile mitochondrial pools. J Peripher Nerv Syst 5: 3-10.

Dhillon RS, Schulte PM (2011) Intraspecific variation in the thermal plasticity of mitochondria in killifish. J Exp Biol 214: 3639-3648.

Duchen MR (2004) Mitochondria in health and disease: perspectives on a new mitochondrial biology. Mol Aspects Med 25: 365-451.

Elgass K, Pakay J, Ryan MT, Palmer CS (2012) Recent advances into the understanding of mitochondrial fission. Biochim Biophys Acta 1833: 150-161.

Escobar-Henriques M, Anton F (2013) Mechanistic perspective of mitochondrial fusion: tubulation vs. fragmentation. Biochem Biophys Acta 1833: 162-175.

Gupta BB, Spessert R, Vollrath L (2005) Molecular components and mechanism of adrenergic signal transduction in mammalian pineal gland: regulation of melatonin synthesis. Indian J Exp Biol 43: 115-149.

Ho AK, Chik CL (1990) Post receptor mechanism in dual receptors regulation of second messengers in rat pineal gland. Prog Clin Biol Res 342: 139-145.

Hori S, Kuroda Y, Saito K, Ohotani S (1976) Subcellular localization of tryptophan-5-mono-oxygenase in bovine pineal glands and raphe nuclei. J Neurochem. 27: 911-914.

Karasek M, Zielinska A, Marek K, Swietoslawski J (2002) Effect of superior cervical ganglionectomy on the ultrastructure of pinealocytes in the Djungarian hamster (Phodopus sungorus): quantitative study. Neuro Endocrinol Lett 23: 443-446.

Kus I, Sarsilmaz M, Ozen OA, Turkoglu AO, Pekmez H, Songur A, Kelestimur H (2004) Light and electron microscopic examination of pineal gland in rats exposed to constant light and constant darkness. Neuro Endocrinol Lett 25: 102-108.

Kuzmicic J, Del Campo A, López-Crisosto C, Morales PE, Pennanen C, Bravo-Sagua R, Hechenleitner J, Zepeda R, Castro PF, Verdejo HE, Parra V, Chiong M, Lavandero $S$ (2011) Mitochondrial dynamics: a potential new therapeutic target for heart failure. Rev Esp Cardiol 64: 916-923.

Larsen S, Nielsen J, Hansen CN, Nielsen LB, Wibrand F, Stride N, Schroder HD, Boushel R, Helge JW, Dela F, Hey-Mogensen M (2012) Biomarkers of mitochondrial content in skeletal muscle of healthy young human subjects. J Physiol 590: 3349-3360.

Lewczuk B (2002) Mechanism of adrenergic regulation of melatonin secretion in the pig pineal gland - in vitro study. 1st ed., University of Warmia and Mazury, Olsztyn.
Lewczuk B, Nowicki M, Prusik M, Przybylska-Gornowicz B (2004) Diurnal rhythms of pinealocyte ultrastructure, pineal serotonin content and plasma melatonin level in the domestic pig. Folia Histochem Cytobiol 42: 155-163.

Lewczuk B, Przybylska-Gornowicz B (1997) Effects of sympathicolytic and sympaticomimetic drugs on pineal ultrastructure in the domestic pig. J Pineal Res 23: 198-208.

Lewczuk B, Przybylska-Gornowicz B (2000a) The effect of continuous darkness and illumination on the function and the morphology of the pineal gland in the domestic pig. Neuro Endocrinol Lett 21: 293-299.

Lewczuk B, Przybylska-Gornowicz B (2000b) The effect of exogenous melatonin on the pinealocyte ultrastructure in the domestic pig (Sus domesticus) depends on the time of its administration. Pol J Vet Sci 3: 29-38.

Lewczuk B, Przybylska B, Wyrzykowski Z (1994) Distribution of calcified concretions and calcium ions in the pig pineal gland. Folia Histochem Cytobiol 32: 243-249.

Lewczuk B, Ziółkowska N, Prusik M, Przybylska-Gornowicz $B$ (2014) Adrenergic activation of melatonin secretion in ovine pineal explants in short-term superfusion culture occurs via protein synthesis independent and dependent phenomena. Biomed Res Int 2014: 715708.

Madhu N, Manna CK (2010) Pineal-adrenal interactions in domestic male pigeon exposed to variable circadian light regimes and exogenous melatonin. Endocr Regul 44: 121-127.

Mironov SL (2006) Spontaneous and evoked neuronal activites regulate movements of single neuronal mitochondria. Synapse 59: 403-411.

Mironov SL (2007) ADP regulates movements of mitochondria in neurons. Biophys J 92: 2944-2952.

Muller M, Mironov SL, Ivannikov MV, Schmidt J, Richter DW (2005) Mitochondrial organization and motility probed by two-photon microscopy in cultured mouse brainstem neurons. Exp Cell Res 303: 114-127.

Nowicki M, Lewczuk B, Przybylska-Gornowicz B (2002) Influence of 4-day long treatment with vasoactive intestinal peptide on ultrastructure and function of the rat pinealocytes in organ culture. Folia Histochem Cytobiol 40: 9-16.

Otera H, Ishihara N, Mihara K (2013) New insights into the function and regulation of mitochondrial fission. Biochim Biophys Acta 1833: 1256-1268.

Otera H, Mihara K (2011) Molecular mechanisms and physiologic functions of mitochondrial dynamics. J Biochem 149: 241-251.

Oxenkrug GF, McIntyre IM, Requintina PJ, Duffy JD (1991) The response of the pineal melatonin biosynthesis to the selective MAO-A inhibitor, clorgyline, in young and middle-aged rats. Prog Neuropsychopharmacol Biol Psychiatry 15: 895-902.

Palmer CS, Osellame LD, Stojanoski D, Ryan MT (2011) The regulation of mitochondrial morphology: intricate mechanisms and dynamic machinery. Cell Signal 23: $1534-1545$.

Pennanen C, Parra V, López-Crisosto C, Morales PE, Del Campo A, Gutierrez T, Rivera-Mejtas P, Kuzmicic J, Chiong M, Zorzano A, Rothermel BA, Lavandero $S$ (2014) Mitochondrial fission is required for cardiomyocyte hypertrophy mediated by a $\mathrm{Ca}^{2+}$-calcineurin signaling pathway. J Cell Sci 127: 2659-2671. 
Perkins GA, Ellisman MH (2007) Mitochondrial architecture and heterogeneity. In: Gibson GE, Dienel GA (eds) Handbook of neurochemistry and molecular neurobiology. Integration of molecular and cellular processes. Springer-Verlag, Berlin, pp 261-295.

Peterson YK, Cameron RB, Wills LP, Trager RE, Lindsey CC, Beeson CC, Schnellmann RG (2013) $\beta_{2}$-adrenoceptor agonists in the regulation of mitochondrial biogenesis. Bioorg Med Chem Lett 23: 5376-5381.

Piquereau J, Caffin F, Novotova M, Lemaire C, Veksler V, Garnier A, Ventura-Clapier R, Joubert F (2013) Mitochondrial dynamics in the adult cardiomyocytes: which roles for a highly specialized cell? Front Physiol 4: 102.

Przybylska-Gornowicz B, Lewczuk B, Ciesielska-Myszka L, Wyrzykowski Z (1994) Cytochemical localization of monoamine oxidase in the pig pineal gland. Folia Histochem Cytobiol 32: 161-166.

Przybylska-Gornowicz B, Lewczuk B, Prusik M, Kalicki M, Ziółkowska N (2012) Morphological studies of the pineal gland in the common gull (Larus canus) reveal uncommon features of pinealocytes. Anat Rec (Hoboken) 295: 673-685.

Przybylska B, Lewczuk B， Wyrzykowski Z， Karasek $M$ (1994) Effects of p-chlorophenylalanine, amiflamine and melatonin treatment on the ultrastructure of pinealocytes in Sus scrofa. Cytobios 77: 233-246.

Reddy PH, Reddy TP, Manczak M, Calkins MJ, Shirendeb $\mathrm{U}$, Mao P (2011) Dynamin-related protein 1 and mitochondrial fragmentation in neurodegenerative diseases. Brain Res Rev 67: 103-118.

Redondo E, Regodon S, Franco A, Masot J, Ghzquez A, Cardinali DP (2003) Day-night changes in plasma melatonin levels, synaptophysin expression and ultrastructural properties of pinealocytes in developing female sheep under long and short photoperiods. Histol Histopathol 18: 333-342.

Robinson MM, Richards JC, Hickey MS, Moore DR, Philips SM, Bell C, Miller BF (2010) Acute (beta) - adrenergic stimulation does not alter mitochondrial protein synthesis or markers of mitochondrial biogenesis in adult men. Am J Physiol Regul Integr Comp Physiol 298: R25-33.
Rube DA, van der Bliek AM (2004) Mitochondrial morphology is dynamic and varied. Mol Cell Biochem 256-257: 331-339.

Sasaki S (2010) Determination of altered mitochondria ultrastructure by electron microscopy. Methods Mol Biol 648: 279-290.

Simonneaux V, Ribelayga C (2003) Generation of the melatonin endocrine message in mammals: a review of the complex regulations of melatonin synthesis by norepinephrine, peptides and other pineal transmitters. Pharmacol Rev 55: 325-395.

Skulachev VP (2001) Mitochondrial filaments and clusters as intercellular power-transmitting cables. Trends Biochem Sci 26: 23-29.

Sundal S, Sharma S (2007) Ultrastructural findings for the mitochondrial subpopulation of mice skeletal muscle after adrenergic stimulation by clenbuterol. J Physiol Sci 57: 7-14.

Tomanek RJ (1989) Sympathetic nerves modify mitochondrial and capillary growth in normotensive and hypertensive rats. J Mol Cell Cardiol 21: 755-764.

Van Laar VS, Berman SB (2013) The interplay of neuronal mitochondrial dynamics and bioenergetics: implications for Parkinson's disease. Neurobiol Dis 51: 43-55.

Weibel ER (1979) Stereological methods. Vol. 1. Academic Press, London.

Wills LP, Trager RE, Beeson GC, Lindsey CC, Peterson YK, Beeson CC, Schnellmann R G (2012) The $\beta 2$-adrenoceptor agonist formoterol stimulates mitochondrial biogenesis. J Pharmacol Exp Ther 342: 106-118.

Wyrzykowski Z, Przybylska B, Wyrzykowska K (1990) The effect of progesterone and progesterone + estradiol on the morphology of the pineal gland in immature female pigs. Z Mikrosk Anat Forsch 104: 265-272.

Wyrzykowski Z, Przybylska B, Wyrzykowska K, Kaleczyc $\mathrm{J}$ (1992) Influence of bilateral ovariectomy on the morphology and ultrastructure of the pineal gland in the pig (Sus scrofa) - quantitative and qualitative studies. Folia Morphol 51: 93-108.

Zielińska A, Swietosławski J, Reiter RJ, Karasek M (2006) The effect of melatonin, $\mathrm{N}$-acetyloserotonin and 6-hydroksymelatonin on the ultrastructure of the pinealocytes of the Syrian hamster (Mesocricetus auratus). Endokrynol Pol 57: 2-6. 